\title{
Validation Web Site: A Combustion Collaboratory over the Internet
}

\author{
Angela Violi ${ }^{1}$, Xiaodong Chen ${ }^{2}$, Gary Lindstrom ${ }^{2}$, Eric Eddings ${ }^{1}$, \\ and Adel F. Sarofim ${ }^{1}$ \\ ${ }^{1}$ Department of Chemical and Fuels Engineering, University of Utah \\ 84112 Salt Lake City, Utah \\ ${ }^{2}$ School of Computing, University of Utah \\ 84112 Salt Lake City, Utah
}

\begin{abstract}
The Soot Model Development Web Site (SMDWS) is a project to develop collaborative technologies serving combustion researchers in DOE national laboratories, academia and research institutions throughout the world. The result is a shared forum for problem exploration in combustion research. Researchers collaborate over the Internet using SMDWS tools, which include a server for executing combustion models, web-accessible data storage for sharing experimental and modeling data, and graphical visualization of combustion effects. In this paper the authors describe the current status of the SMDWS project, as well as continuing goals for enhanced functionality, modes of collaboration, and community building.
\end{abstract}

\section{Introduction}

The emergence of the Internet has enabled interactions among people working in the same field around the globe. This has inspired many efforts to address the associated problems of effective group communication, and provide access to information sources that are geographically and administratively disparate. Collaboratories are one way to provide virtual shared workspace to users. A Collaboratory is a wide area distributed system supporting a rich set of services and tools that facilitate effective synchronous and asynchronous communication between two or more people who are not co-located [1]. To date, collaboratories have been developed in many areas including material science [2], natural science [3], telemedicine [4], space physics [5], and environmental molecular sciences [6]. In the combustion field, web-based collaboratories have been developed in the areas of turbulent diffusion flames [7] and diesel engines [8].

V.N. Alexandrov et al. (Eds.): ICCS 2001, LNCS 2074, pp. 485-493, 2001.

(C) Springer-Verlag Berlin Heidelberg 2001 
The University of Utah Center for the Simulation of Accidental Fires and Explosions (C-SAFE) [9] is an interdisciplinary project funded under the DOE Accelerated Strategic Computing Initiative Alliances program. This center focuses on providing largescale high performance science-based tools for the numerical simulation of accidental fires and explosions, targeted at issues associated with safe handling and storage of highly flammable materials.

The Validation Web Site (SMDWS) [10] is in turn a research project under C-SAFE providing a shared problem exploration environment for combustion researchers. The SMDWS enables researchers to work together across geographic and organizational boundaries to solve complex interdisciplinary problems, and to securely share access to resources at participating web including specialized databases and computational servers. In short, the goal of the SMDWS project is to make science and information exchange more efficient, opportunistic and synergistic for combustion researchers at large.

This paper discusses the application area, the SMDWS requirements, and the SMDWS architecture and design.

\section{Application: Combustion Modeling}

Soot formation is a significant environmental problem associated with the operation of many practical combustion devices.

An accurate simulation of soot formation is critical to the C-SAFE goal of predicting the radiative heat transfer in fires, since soot is a dominant component of hydrocarbon fires. High heat flux and emissions from accidental fires pose significant human and property hazards.

The Soot Model Development Web Site has been developed to allow the C-SAFE group working on the development of soot models to collaborate with researchers at LLNL, LBL, SNLA, SNLL, UC Berkeley, Polytechnic of Milan, and the University of Naples. Model development is a complex task requiring a close interplay among experimentation, development of chemical submodels, model validation and formulation of new modeling concepts. Progress in this already difficult task is further complicated by the interdisciplinary nature of the required collaboration, as well as the geographical distribution of experts in the field throughout the national laboratories, industry, and universities. Effective model development requires validation of individual model components as well as strategies for their integration. For example, C-SAFE's soot modeling task has the specific goal of developing reliable, experimentally validated models of soot formation that can be used in a numerical simulation and optimization of combustion devices. Success in this task clearly requires the critical comparison and co-design of soot formation mechanisms being developed both within and outside of C-SAFE. Hence a coordinate effort between the many researchers is required to obtain the best soot formation mechanism possible. It was judged early in the C-SAFE project that development of an interactive, web-based tool for this purpose was essential to achieving this goal. The C-SAFE Validation group has adopted the role of leading this 
effort and is hosting an electronic interaction facility with the assistance of Computer Scientists within the C-SAFE team. We have collected several combustion modeling codes and made them available to the SMDWS over the Internet: ChemkinII, a chemical kinetics package for the analysis of gas-phase chemical kinetics; ChemkinII/Soot [11], which is Chemkin with kinetic modeling of soot formation; and DSmoke [12], which supports the analysis of different reactors in series or in parallel coupled with a mixer or splitter. Several reaction mechanisms are also available at the SMDWS: Lawrence Livermore National Laboratory's mechanisms for the formation of aromatic compounds and other species $[13,14]$, kinetic mechanisms by Prof. Frenklach's group at the University of California at Berkeley $[15,16]$, reaction schemes by Polytechnic of Milan, Italy [17], etc. The SMDWS also provides links to important public databases in the area (e.g., www.ecs.umass.edu/MBMS/) for browsing and comparison with data contributed to the SMDWS web site. Also provided is a database of premixed flames data collected from the literature. The modeling results obtained by Utah's own soot combustion group are reported on the web to invite critical feedback and solicit additional contributions from other research groups. The goal of the SMDWS is to make doing science, engineering and information exchange among research partners more efficient and synergistic. Researchers are able to tackle new problems with their existing methods and to access new methods through collaborations that were previously infeasible due data and software interchange obstacles.

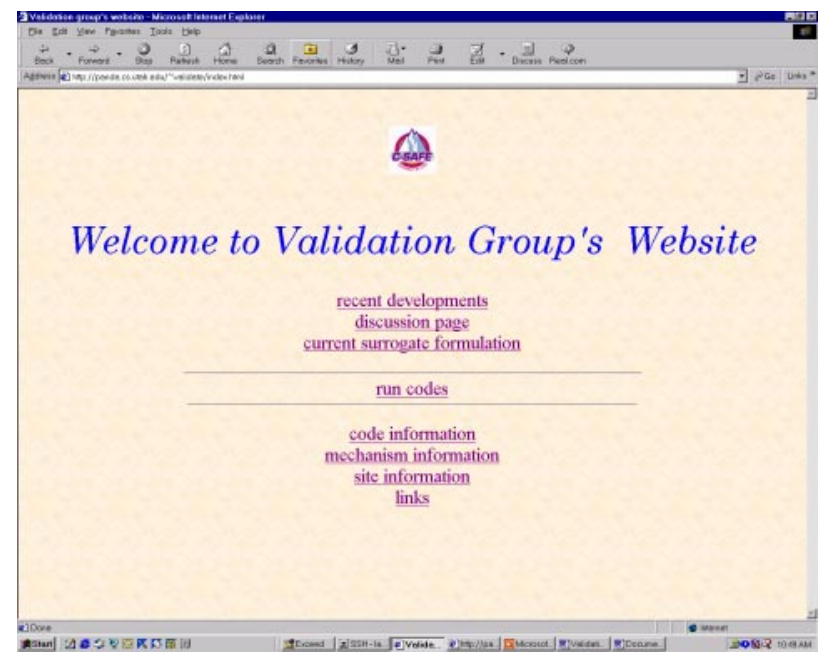

Fig. 1. Soot Model Development Web Site welcoming page 


\section{Soot Model Development Web Site Requirements}

The SMDWS has defined a specific set of requirements in order to accomplish these goals. The researchers at different sites must be able to:

- Share graphical data easily using web browsers

- Discuss modeling strategies and easily share model descriptions between groups

- Archive collaborative information in a web-accessible electronic notebook

- Utilize a remote execution system to run combustion models at remote locations and

- Readily explore and analyze experimental data and modeling results through web browsers

At present a first approximation to these goals has been implemented, including use of our web server as the only computational host, and a rudimentary file based electronic notebook facility. Many of zero-dimensional chemistry simulations (such as Premix of the Chemkin software) can run for hours to days on high-end workstations and produce kilobytes of data. The resulting data sets are visualized using simultaneous 2-D plots of multiple variables. In addition to dealing with the practicalities of such scale issues, many of the collaborative tools (e.g., modeling tools) must be integrated to allow data to flow easily between tools. The SMDWS architecture must support rapid and easy integration of new modeling and visualization tools and newly developed models. Modeling and visualization tools include commercially developed software, as well as software developed by some of the partners, often written in Fortran (for example the DSmoke code developed by Prof. Ranzi's group at the University of Milan, Italy). Users must be able to locate and combine resources and data to form effective teams.

\section{Design of the SMDWS Architecture}

The SMDWS is based on a software framework facilitating the addition and integration of computing services and supporting tools. Careful thought as to the underlying architecture is critical to the smooth evolution of the web site to meet growth and diversification of collaborative, modeling, and visualization demands. Our framework naturally divides into client and server components. The server component has the following properties:

1. Support for running codes from local and remote contributors, including pre- and post-processors

2. Validation of required and optional input

3. Delivery of output files as specified by client 
4. Browsing and query-driven inspection of arbitrarily large output files

5. Return of partial results upon failure, with diagnostic reports.

The SMDWS server architecture consists of two parts: a web server and database server. Both parts run under Window NT Server 4.0.

1. Our web server uses an Apache 1.3.14 web server, supplemented by an Apache JServ 1.0 servlet engine. One multipurpose Java servlet (under JSDK2.0) receives two kinds of requests from clients:

a) Get the input parameters and input data files for simulation run from client side, then call a DOS shell script and an Expect script (Expect 5.21) to run the executable codes, and finally store the results in the database.

b) Receive a database query from client side, then get the database response and return the results to the client. Client web pages provide input parameters through a Java Applet (JDK1.1.8) interface. In addition, we have provided an optional username and password protected version of our website where Raven SSL 1.4.1 provides Digest Authentication for web security.

2. Our database server runs on Microsoft SQL Server 7.0. The Java servlet communicates with SQL Server through an RmiJdbc server and a JDBC/ODBC bridge.

The client framework is Java code supporting the integration of contributed combustion modeling codes (rarely in Java) to be integrated into the SMDWS. Properties include:

- Status monitoring of long running execution requests with periodic updates

- Termination and subsequent recovery of intermediate results

- Flexible invocation of all computational resources, including pre-processors and post-processors

- Definition and upload of required and optional input files

- Inspection and download of expected output files

- Parameterizable visualization, currently limited to 2D plotting

Employing client and server frameworks allows new capabilities to be added easily, facilitates maintenance of SMDWS client and server code, maximizes code reuse and enables integration with third party components such as web and database server tools. 
Future development of the SMDWS will support electronic notebooks and shared workspaces where researchers can annotate combustion codes, input data sets, and result files --- all accessible through their web browsers. The electronic notebooks will contain a rich variety of information - text and images, and links to information can be simultaneously viewed, edited and annotated on the pages of a notebook. Similarly, it is planned that shared workspaces will be made interactively accessible using a web browser, enabling users to dialog about modeling codes, input files, output files, data plots, and notes on the computational models.

A 2-D plotting package using the Java 2D Graph Package v2.4 has been integrated into the SMDWS modeling client template, such that users can interactively plot modeling results from several modeling runs. Users can then save plots as files or save plot files in a file repository.

\section{Collaborative Tools in the SMDWS}

The challenges involved in successful deployment of the SMDWS include development and implementation of an appropriate collaboratory infrastructure, integration of new interdisciplinary tools, and the deployment and adoption of the SMDWS among the research community. To accomplish this, we have planned a systematic approach to the development, integration, and testing of tools. The initial phase of deployment has been to directly involve the most interested combustion researchers in tool development and testing. The SMDWS has been found to have highest impact when geographically separated researchers are motivated to interact directly with each other to achieve their goals. The focus effort provides direct support to researchers as they confront some of the cultural changes inherent in electronic collaboration. These changes include the use of new technologies and the development of social behaviors in electronic communication and collaboration [1]. One challenge in particular is the balance of the increased access to each other data, resources, and presence with the desire to invoke electronic security to protect the same.

The SMDWS project will be broadening the target research to multiple projects including a broader range of collaborators and the dissemination of new technology to the other interested groups. The broadening of the target research areas will provide a greater test of the generality of our approach and an opportunity to incorporate some advanced tools.

\subsection{Examples of SMDWS Application}

Descriptions of illustrative scenarios in the SMDWS serve to further describe its application in combustion research. Here we provide a few brief descriptions: 
- A user can retrieve previous results generated by a collaborator from a shared workspace, interpret this data then rerun modeling code with new inputs, and put new results in the shared workspace that can be viewed by collaborators.

- A scientist can start a modeling run in collaboration with a distant colleague. The results will be stored in a repository for both to see.

- The combustion scientists upload input files from local machines for remote execution of the code using the respective SMDWS clients. Output files are returned to the scientist's machines and the modeling results could be immediately analyzed using the SMDWS client's interactive 2D plotting package (see Figure 2).

All the calculations are documented in web-based files that can be accessed by collaborators. As mentioned above, our ultimate goal is for the combustion scientist to use real-time collaboration tools to discuss results with colleagues.
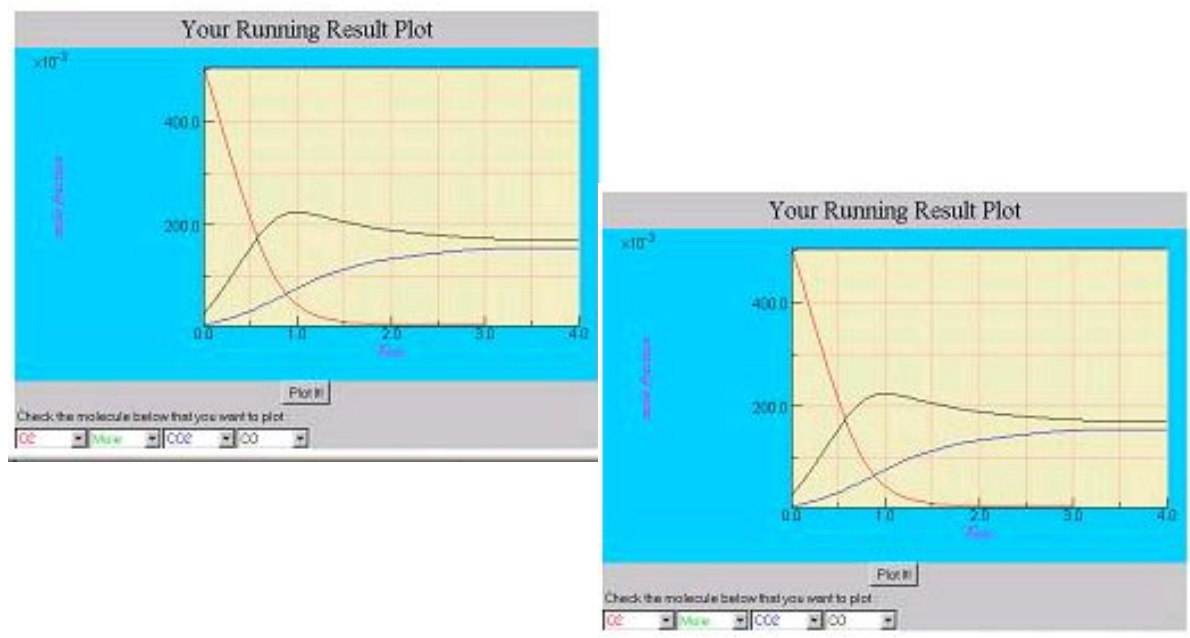

Fig. 2. Calculation results for some species concentrations

\section{Status and Future Work}

The SMDWS project is continuing to develop collaborative technologies for combustion researchers distributed throughout the national laboratories and academia. We have assembled an overall soot formation mechanism starting from a surrogate hydrocarbon fuel that has been experimentally developed to reproduce the features of jet fuels important for pool fires. This mechanism draws on experimental and ab initio 
approaches to obtaining rate data for the elementary reaction assembled from the literature. Validation of the mechanism has been performed by comparison of the soot formation mechanism with measurements from laboratory flames. Thus for the site permits the remote running of the soot models on a local server, thus providing a means for all collaborators work with the same version of mechanisms and models. The site also provides a Discussion Page for dialogue on the results of mechanism comparisons. User friendly and low maintenance security is a difficult challenge and will require continuous development with user feedback for some time. In the future we expect to offer a real-time conferencing capability. Ultimately, desktop video and data conferencing among researchers will be investigated as appropriate Internet connection bandwidth becomes available.

Collaboratory technology is having an impact, however. One of the most significant impacts of the SMDWS is to extend the researcher's capabilities to use new tools by making possible the use of such tools in collaboration with an expert at another location. The ability to handle such knowledge resources is a key to interdisciplinary collaboration and an important characteristic of the SMDWS.

Future work will build on the current successes in framework implementation, tool development. We are searching simultaneously both a greater range of capabilities and a higher level of user friendliness. New target research groups will involve international participation with new challenges for interoperability and bandwidth. We anticipate new client/server templates for multi-code simulations, new capabilities for collaborative visualization and group collaboration built on new tools, and improved commercial tools.

\section{Acknowledgement}

The scope of the work addressed in this project would not be possible without the efforts, support of the group of people associated with the C-SAFE, the School of Computing at the University of Utah, Dr. Westbrook's group at LLNL, Prof. Frenklach at University of California, Prof. D'Alessio at the University of Naples, Italy and Prof. Ranzi at the University of Milan, Italy.

This research is funded by the University of Utah Center for the Simulation of Accidental Fires and Explosions (C-SAFE), funded by the Department of Energy, Lawrence Livermore National Laboratory, under subcontract B341493. Additional support was provided by the University of Naples "Federico II", Department of Chemical Engineering.

\section{References}

1. Kouzes R.T., Myers J.D. and Wulf W.A., Collaboratories: Doing Science On the Internet, IEEE Computer, volume 29, number 8, August 1996 
2. Parvin B., Taylor J. and Cong G., Deep view: a collaborative framework for distributed microscopy, Proceedings of SC 98, Orlando Florida, Nov.1998

3. Sunderam V.S., Cheung S.Y., Hirsh M. et al., CCF: collaboratory computing frameworks, Proceedings of SC 98, Orlando Florida, Nov.1998

4. Kilman D.G. and Forslund D.W., "An international collaboratory based on virtual patient records, Communications of the ACM, volume 40, n.8, (1997)

5. Subramanian S., Malan G.R., Sop Shim H. et al., "Software Architecture for the UARC web-based collaboratory", IEEE Internet Computing, volume 3, n.2, (1999), pg. 46-54

6. Payne D.A. and Myers J.D., The EMSL collaborative research environment (CORE) collaboration via the World Wide Web, Proceedings of the Fifth Workshop on Enabling Technologies: infrastructure for collaborative enterprises (WET ICE’96), Stanford, California, June 1996

8. http://www.ca.sandia.gov/tdf/Workshop.html

8. http://www-collab.ca.sandia.gov/dcc/

9. http://www.C-SAFE.utah.edu/

10. http://panda.cs.utah.edu/ validate

11. Kenneth L. Revzan, Nancy J. Brown and Michael Frenklach, at http://www.me.berkeley.edu/soot/

12. Ranzi E., Faravelli T., Gaffuri P. and Sogaro A., Combustion and Flame 102: (1995), 179192

13. Marinov, N.M., Pitz, W.J., Westbrook, C.K., Castaldi, M.J., and Senkan, S.M., Combustion Science and Technologies, 116-117, (1996), 211-287

14 Curran, H. J., Gaffuri, P., Pitz, W. J., and Westbrook, C. K., Combustion and Flame 114, (1998), 149-177

15. Hai Wang and Michael Frenklach, Combustion and Flame 110, (1997), 173-221

16. Joerg Appel, Henning Bockhorn, and Michael Frenklach, Combusion and Flame 121, (2000), 122-134

17. Ranzi E., Sogaro A., Gaffuri P., Pennati G., Westbrook C.K., Pitz W.J., Combustion and Flame 99, (1994), 201-211 